\title{
1C: Non-gastric diseases related to H. pylori infections
}

\section{C:01 HELICOBACTER PYLORI AND REFLUX OESOPHAGITIS}

R.J.L.F. Loffeld, B.F.M. Werdmuller. Department of Internal Medicine, Ziekenhuis De Heel, Zaandam, The Netherlands

The main pathogenetic factor in reflux oesophagitis apparently is the reflux of acidic stomach contents. From a theoretical point of view it could be postulated that HP indirectly has an effect on reflux oesophagitis by making the refluxate more aggressive.

A prospective study was done in consecutive patients presenting with reflux disease.

During one year all consecutive patients undergoing upper GI endoscopy were studied for the presence of HP. Inclusion criterion was the presence of hiatal hernia and/or reflux oesophagitis, and Barrett's oesophagus. Exclusion criterion was the presence of oesophageal or gastric cancer and the presence of concomitant peptic ulcer disease or macroscopic gastritis. Biopsy specimens were taken for HE stain, urease test and culture. A serum sample was taken for determination of IgG antibodies against HP using a home-made ELISA. As a reference group patients in whom endoscopy did not show abnormalities were included. Reflux oesophagitis was present in 118 patients $\left(68 \sigma^{\circ}, 50 \%\right.$, mean age 55.3 years). Hiatal hernia without oesophageal inflammation was seen in 109 patients $\left(43 \sigma^{\circ}, 66^{\circ} \%\right.$, mean age 57.3 years), and Barrett's oesophagus in 13 patients $\left(80^{\circ}, 5 \%\right.$, mean age 61.8 years). The reference group consisted of 399 patients (166 $0^{\circ}, 233$ $\odot$, mean age 48.4 years).

HP was present in $27 \%$ of oesophagitis patients, in $23 \%$ of Barrett's oesophagus, in $32 \%$ of hiatal hernia, in $28 \%$ of oesophagitis with hiatal hernia and $25 \%$ of oesophagitis without hiatal hernia, and in $51 \%$ of controls. Prevalence of HP was significantly lower in all groups compared if with the reference group $(p<0.001)$. There was no difference between the different groups. Patients with oesophagitis and HP were significantly older than oesophagitis patients without HP, 61.5 years (SD 17) versus 53 years (SD 17) $(p<0.001)$. The same was true if helicobacter positive and negative references were compared, 52 years (SD 17) versus 44.8 years (SD 17.6) $(p<0.0001)$. There was no difference in age between the different grades of oesophagitis.

It is concluded that the prevalence of HP in patients with reflux disease is significantly lower than in a reference group with normal oesophagus. The patients with reflux oesophagitis and HP are older than those without the infection indicating that the age cohort effect is present. In case of reflux oesophagitis it is essential to know whether the patients is helicobacter positive, because long-term acid suppressive therapy will lead to atrophic gastritis. HP has no direct or indirect role in the pathogenesis of reflux oesophagitis.

\section{C:02 WHAT IS THE INFLUENCE OF HELICOBACTER PYLORI INFECTION ON MUCOSAL LESIONS DEVELOPMENT IN PATIENTS TAKING ACETYLSALICYLIC ACID?}

H. Kordecki, M. Kurowski, D. Pilecka, R. Kosik. M. Curie Hospital, Szczecin, Poland

Acetylsalicylic acid (ASA) is one of the drugs widely taken for ischaemic heart disease. But up to now the influence of ASA on gastric and duodenal mucosa is still not known. Recently published reports suggest that repeated doses of ASA increase mucosal tolerance and ASA could cause the mucosal injury only during the first days of treatment. However these studies were performed in rats.

The aim of our study was to evaluate the frequency of mucosal lesions (m.l.) in the upper digestive tract as well as the frequency of Helicobacter pylori (H.p.) infection in patients chronically receiving acetylsalicylic acid in the doses ranged from 70-300 mg daily. ASA was administrated for prevention of ischaemic heart disease attacks in 60 patients treated in the Department of Cardiology in our hospital.

These patients were 31-72 years old (mean $50.5 \mathrm{y}$ ). They were sent for endoscopic examination of upper digestive tract because of the planned heart surgery. Non of them reported any complaints from the upper abdomen.

The control group comprised the same number of patients of similar age treated for ischaemic heart disease, who did not take ASA due to various contrindications.

In $52(87 \%)$ patients suffering from ischaemic heart disease and taking ASA the presence of mucosal erosions in the stomach (some of them were haemorrhagic) and/or in the duodenal bulb were disclosed. $12(20 \%)$ patients had gastric ulcer and the microscopical examination revealed active gastritis in $39(65 \%)$ patients. $76 \%$ of patients receiving ASA were infected with H.p. (positive rapid urease test confirmed by microscopical examination). In the control group comprised patients not taking ASA only in 4 patients (7\%) the presence of single mucosal erosions was revealed. 54 patients had no changes in the upper digestive tract. The presence of H.p. was disclosed in $16(27 \%)$ patients.

The study we performed failed to confirm the views that long-term ASA therapy is free of gastric and duodenal m.l. Although the study group was relatively small we cannot share the opinion that gastric and duodenal ulcers occurring during ASA therapy are not hazardous for patients. However it should be stressed that significantly higher percentage of H.p. infection appears in patients treated with ASA in comparison with control group, what in our opinion is favouring factor of m.l. development.

\section{C:03 CHANGES IN ANTIBODIES TO HELICOBACTER PYLORI (HP) AND IN JOINT MANIFESTATIONS IN PATIENTS WITH RHEUMATOID ARTHRITIS (RA) AFTER ERRADICATION FROM HP. PRELIMINARY RESULTS}

L.B. Graff ${ }^{1}$, L.P. Andersen ${ }^{2}$, A. Bremmelgaard ${ }^{1}$, O. Bonnevie ${ }^{\text {' }}$ S. Bondesen ', A. Gernow ' , A. Savnik ${ }^{1}$, J. Blom ${ }^{3}$, N. Høiby ${ }^{2}$, B. Danneskiold-Samsøe '. ' Frederiksberg Hospital, ${ }^{2}$ Rigshospitalet, ${ }^{3}$ Statens Seruminstitut, Copenhagen, Denmark

Aim: To evaluate the effect of erradication from $H P$ in patients with RA with regard to IgG antibodies and to joint manifestations.

Methods: 54 RA patients participate in the study, 17 have been found $H P$-culture positive and 8 patients, 6 women and 2 men, median age 62.5 years $(39-79$ y.) and median disease duration 12.5 years $(2-35$ y.) have been followed for 18 weeks. The patients were examined for IgG antibodies (LMW IgG) to $H P$ by ELISA before and after erradication from $H P$. Biopsies were taken and examined according to the Sydney classification of gastritis before and 6 weeks after ended antibiotic treatment. The patients' joints were examined for pain at palpation and for swelling and the joints were giving scores from 0 to 3 in each category. The serological and the clinical examination were performed before treatment, 6 and 18 weeks after.

Results: Median LMW-IgG was before treatment $284.5 \mathrm{Eu}(146-840$ Eu), after 6 weeks $163 \mathrm{Eu}(119-832 \mathrm{Eu})$ and after 18 weeks $97 \mathrm{Eu}(0-374)$, $\left(\mathrm{p}_{6 \text { weeks }}=0.02\right)$, $\left(\mathrm{p}_{18 \text { weeks }}<0.01\right)$. The median score of joint pain was 30 (4-93) before treatment, $28(2-112)$ after 6 weeks and $34.5(3-107)$ after 18 weeks $\left(p_{6 \text { weeks }}=0.84\right),\left(p_{18 \text { weeks }}=0.09\right)$. The median score of joint swelling was $7(2-21)$ before treatment, $2(0-40)$ after 6 weeks and 4 $(0-23)$ after 18 weeks $\left(p_{6 \text { weeks }}=0.20\right),\left(p_{18 \text { weeks }}=0.32\right)$.

Conclusion: A significant change was found in IgG to $H P$ after erradication ( $p<0.01)$, but the changes in joint pain and joint swelling showed no significance $\left(\mathrm{p}_{\text {pain }}=0.09\right)$, $\left(\mathrm{p}_{\text {swelling }}=0.32\right)$.

\section{C:04 HP PREVALENCE IN IBD PATIENTS: ROLE OF IBD TREATMENT}

M. De Reuck, M. Brad, J. Otero, A. Cozzoli, P. Denis, E. De Koster, M. Deltenre. Brugmann University Hospital, Brussels, Belgium

A lower prevalence of HP in IBD patients (pts), attributed to sulfapyridine (SP) treatment, has been suggested (El Omar Gut 1994; 35: 1385-8). We determined HP prevalence in 130 IBD pts: 85 Crohn's disease (CD), 45 ulcerative colitis (UC), using serology (78), Breath Test (21), histology (31). IBD treatment: none (NT), enteric coated SP, 5-ASA. We compared with 155 Non Ulcer Dyspepsia (NUD) pts. Results: HP prevalence is shown in Table 1.

Prevalence of HP in IBD $(23 \%)$ lower than in NUD $(47 \%) p<0.0001$. No difference CD (19\%)-UC (31\%) (ns). UC NT (46\%) not different from NUD $(47 \%)$, higher than treated UC $(14 \%, p<0.05)$ or treated CD $(18 \%$, $\mathrm{p}<0.01)$. No difference SP $(16 \%)$ to 5-ASA (18\%). Untreated CD (23\%) not significantly lower than untreated UC (46\%) (ns). Conclusions: We confirm the lower HP prevalence in treated IBD patients, both CD and UC. HP prevalence in untreated UC patients is not different from NUD patients. HP prevalence in untreated $C D$ patients may be lower than in untreated UC patients, but our series is too small to reach a statistically significant difference. We find no difference in HP prevalence between pts treated with 


\begin{tabular}{llrrl} 
Table 1 & \multicolumn{3}{l}{} \\
\hline & & $\mathrm{N}$ & $\mathrm{HP}+$ & $\% \mathrm{HP}+$ \\
\hline NUD & & 155 & 73 & 47 \\
CD & NT & 13 & 3 & 23 \\
& SP & 22 & 5 & 23 \\
& 5-ASA & 50 & 8 & 16 \\
& all CD & 85 & 16 & 19 \\
UC & NT & 24 & 11 & 46 \\
& SP & 9 & 0 & 0 \\
& 5-ASA & 12 & 3 & 25 \\
& all UC & 45 & 14 & 31 \\
All IBD & & 130 & 30 & 23 \\
\hline
\end{tabular}

enteric-coated SP or 5-ASA. These data suggest that IBD treatment (SP or 5-ASA), and maybe CD per se, is associated with a lower HP prevalence.

\section{C:05 EFFICACY OF STANDARD TRIPLE THERAPY IN THE ERADICATION OF H. PYLORI INFECTION IN PATIENTS WITH CORPORAL ATROPHIC GASTRITIS (CAG)}

B. Annibale, M. Marignani, M. Paoletti, S. Angeletti, G. Antonelli, M.R. Aprile ', G. D'Ambra, C. Bordi ' ', G. Delle Fave. University "La Sapienza" Roma Italy; ' University "La Sapienza" Parma Italy

CAG is a condition characterized by atrophy of oxyntic mucosa hypo/achlorydria and fasting hypergastrinemia. It has recently been observed that a significant proportion of CAG patients are Hp infected and that Hp infection is significantly associated with the development of corporal atrophy in patients treated long-term with proton pump inhibitors. No studies until now have been specifically addressed to prospectively investigate in CAG patients, if it is possible to treat the infection since the lack of acid secretion could determine less favourable condition to his eradication. 19 consecutive patients (15 F, 4 M aged 34-72) with Hp positive CAG (histology, culture, Ig G; at least two of these tests positive) were treated for 4 wks with bismuth subsalicilate 240 b.i.d.; amoxicilline $1 \mathrm{~g}$. tid post-prandially and metronidazole $250 \mathrm{mg}$ tid post-prandially were given during the first 2 wks of therapy. Endoscopy was again performed after 6 mos, to evaluate Hp status by histology and colture in antral $(n=5)$ and body biopsies $(n=8)$. The gastritis status was graded according to the Sydney system. Fasting gastrin levels ( $\mathrm{pg} / \mathrm{ml}$; specific RIA) and title of IgG Hp (U/; Elisa GAP test, Biorad) were also determined. Results are expressed as median (range), non-parametric test for paired data was used for statistical evaluation. Results: Overall eradication rate was $78.9 \%$ $(15 / 19 \mathrm{pts})$. Minor side effects were recorded in about half of patients, but none determined therapy withdrawn. Gastrin levels decreased dramatically in eradicated patients [ $220(49-1400)$ vs $42(10-285) ; p<0.001]$ as IgG Hp [80 (10-100) vs $31(0-65) ; \mathrm{p}<0.005]$ Corporal chronic inflammation and atrophy were significantly reduced after eradication [respectively: 2 $(1-3)$ vs $1(0-2) ; p=0.001$ and $2(1-3)$ vs $1(0-3) ; p=0.031$ ]. Inflammation activity completely regressed $[1(0-3)$ vs $0(0-0)]$. Conclusions: These data show that is possible to efficaciously treat $\mathrm{Hp}$ infection in CAG patients, determining a pronunced reduction of gastrin levels and a significative reduction in the score of corporal chronic gastritis.

\section{C:06 DOES HELICOBACTER PYLORI INFECTION HAVE A ROLE IN PERNICIOUS ANEMIA?}

G.I. Pérez-Pérez, R. Carmel, M.J. Blaser. Vanderbilt University Department of Veterans Affairs Medical Center, Nashville TN and University of Southern California Los Angeles CA

Previous studies indicate that patients with pernicious anemia (PA) are infected with $H$. pylori $(\mathrm{Hp})$ less often than are age-matched controls. However, $\mathrm{Hp}$ infection may be present prior to the development of PA and gradually become absent due to the development of the associated type A atrophic gastritis. To determine whether evidence of $\mathrm{Hp}$ infection may disappear during the course of the illness, we studied 47 PA patients (mean age $58.2 \mathrm{y}$ and $36.2 \%$ male) prospectively. The diagnosis of PA was established by either: i) abnormal Schilling test corrected by intrinsic factor (IF); ii) lack of IF in stimulated gastric juice or iii) the combination of low $B_{12}$ levels and serum anti-IF antibodies. Sera were available for a mean of $5.9 \pm 0.5$ years and ELISAs were used to detect $\mathrm{Hp}$-specific serum IgG or IgA. In total, 12 (25.5\%) patients showed evidence of $\mathrm{Hp}$ infection. Comparison of the $12 \mathrm{Hp}$-seropositive $(\mathrm{Hp}+)$ and $35 \mathrm{Hp}$ seronegative $(\mathrm{Hp}-)$ PA patients showed that the former group had significantly lower gastrin (mean $686 \mathrm{vs} 1293 \mathrm{pg} / \mathrm{ml}, \mathrm{p}=0.045$ ) and higher pepsinogen $\mathrm{A}$ levels (mean 27.1 vs. $11.6 \mathrm{pg} / \mathrm{ml}, \mathrm{p}=0.05$ ), factors consistent with less severe atrophic gastritis. The $35 \mathrm{Hp}-$ patients were followed for a total of 204 person-years; none showed seroconversion. The $12 \mathrm{Hp}+$ were followed for a total of 63 person-years, (mean 5.25 years); during this time, 4 (33\%) of the patients seroreverted. Thus, among patients with PA, the seroreversion rate was greater than $6 \%$ per year. Those who seroreverted were younger (52 vs. $69 \mathrm{y}, \mathrm{p}=0.06$ ) and had lower pepsinogen $\mathrm{A}$ levels (9 vs. $39, \mathrm{p}$ $=0.057$ ) than those remaining positive. These data are consistent with the hypothesis that $\mathrm{Hp}$-infection precedes at least some cases of PA and that the infection reverses with time; and presumably with progressive gastritis. This pattern may be especially associated with a subset of PA characterized by less severe gastrin and pepsinogen abnormalities in infected persons. The development of atrophic gastritis as part of PA leads to gradual elimination of the organism and failure to detect a past $\mathrm{Hp}$ infection. Prospective studies, in the pre-PA stage of gastritis are needed to clarify the putative role of $\mathrm{Hp}$ in causing PA.

\section{C:07 ROLE OF H. PYLORI INFECTION IN PEPTIC ULCERATIONS} IN 50 PATIENTS WITH CIRRHOSIS

L. Prigent-Delecourt, D. Lamarque, F. Roudot-Thoraval, R. Akremi, M.T. Chaumette, J.P. Richardet, J.C. Delchier. Hopital Henri Mondor 94000 Créteil, France

The pathogenesis of gastric and duodenal ulcerated mucosal lesions in patients with cirrhosis remains unclear. The aim of this prospective study was to determine the pathogenic factors associated with peptic ulcerations in patients with cirrhosis.

Patients with histologically proven cirrhosis and not recently treated by antibiotics, antisecretory or anti-inflammatory drugs, were enrolled and referred for upper gastrointestinal endoscopy. Upper digestive tract bleeding within the last week was an exclusion criteria. Age, gender, smoking habit, recent alcohol intake, etiology of cirrhosis, Child-Pugh grade were recorded and basal gastrinemia was determined. Esophageal varices were graded from 0 to 3 . Severity of hypertensive gastropathy in the body and the antrum was graded from 0 to 3 for erythema, edema and snake-skin mosaic pattern (maximum score: 18). H. pylori status was determined from rapid urease test and histology on biopsy samples or 13-C urea breath test.

Fifty patients were included. There were 42 males and 8 females, mean age \pm SD: $56 \pm 11$ years. Cirrhosis was alcoholic in 35 , grade A, B and C in the Child-Pugh classification in 13,18 and 19 respectively. Twenty seven (54\%) were $H p$ positive. Peptic ulcerations were present in $18(36 \%)$ : gastric ulcer in 7, duodenal ulcer in 7, gastric erosions in 5, duodenal erosions in 3. Univariate analysis showed that peptic ulcerations were not significantly related with age, gender, smoking habit, etiology of cirrhosis, Child-Pugh grade, esophageal varices grade, $H p$ positivity $(8 / 18$ vs $19 / 32$, $p$ $=0.47$ ), and basal gastrinemia. Univariate and multivariate analysis showed that they were significantly related to a high hypertensive gastropathy score $(10.7 \pm 4.4 v s 7.1 \pm 4.4, \mathrm{p}<0.02)$ and to a recent alcohol ingestion $(67 \%$ vs $29 \%, \mathrm{p}<0.01$ ).

Conclusion: Peptic ulcerations in cirrhotics are unrelated to $H p$ despite high prevalence of infection. They are significantly and independently related to hypertensive gastropathy severity and to recent ingestion of alcohol.

\section{C:08 CORRELATION BETWEEN GASTRIC INFECTION WITH HELICOBACTER PYLORI AND PLASMA LEVELS OF FIBRINOGEN, PLASMINOGEN ACTIVATOR INHIBITOR (PAI) AND VON WILLEBRAND FACTOR (VWF) ANTIGEN}

L. Bierti, C. Cernuschi, C. Abbiati, G. Beccari, R. Di Battista, R. Marchi, A. Federici ${ }^{1}$, B. Bottasso ${ }^{1}$, N. Di Rocco ${ }^{1}$, R. de Franchis,

P.M. Mannucci ${ }^{1} .^{1}$ Gastroenterology service, University of Milan, IRCCS Ospedale Policlinico, Milan, Italy; A. Bianchi Bonomi Hemophilia and Thrombosis Centre, University of Milan, IRCCS Ospedale Policlinico, Milan, Italy

There is evidence suggesting that patients at risk for coronary heart disease (CHD) are more likely to suffer from Helicobacter Pylori (HP) gastric infection than controls. In addition, preliminary data suggest that patients with HP infection may have high levels of plasma fitrinogen Aim: to determine whether HP infection is associated with increased plasma levels of fibrinogen and other risk factors for CHD such as PAI and vWF antigen. Methods: consecutive patients undergoing upper GI endoscopy at our Institute were studied. HP infection was diagnosed by histological (modified Giemsa) and biochemical (CLO-test) methods. Concomitant inflammatory conditions were excluded by clinical examination and by measuring ESR WBC, PCR, alpha-1-acid-glycoprotein. Fibrinogen levels were measured by Clauss' technique, while vWF antigen and PAI were assayed by ELISA. Statistical analysis was carried out by means of Student's $t$ test. Results: 130 patients (66 HP positive and $64 \mathrm{HP}$ negative) were enrolled. There was no difference between the two groups in sex, age, smoking history, hormonal therapy in females, arterial hypertension, dyslipidaemia, values of acute phase reactants, family history of CHD. Plasma fibrinogen levels $(\mathrm{mg} / \mathrm{dL})$ were $318 \pm 76$ in the HP+ group vs $291 \pm 65$ in the HP- group $(p=0.037)$; plasma PAI levels (ng/dL) were $41.2 \pm 29$ in $\mathrm{HP}+$ and $35.8 \pm$ 
24 in HP- patients ( $p=$ N.S.); vWF antigen levels (U/dL) were $148 \pm 53$ in the HP+ group vs $114 \pm 52$ in the HP-group $(p=0.01)$. Conclusion: Plasma vWF and fibrinogen levels were significantly higher in HP+ than in $\mathrm{HP}$ - patients. PAI levels were also increased in HP+ patients, although the difference did not reach statistical significance. Further studies on larger patients populations are required to clarify whether $\mathrm{HP}+$ patients are indeed at higher risk for $\mathrm{CHD}$.

\section{C:09 REFLUX ESOPHAGITIS: A COMPLICATION OF HELICOBACTER PYLORI ERADICATION THERAPY?}

N. Saccà, A. De Medici, S. Rodinò, M. De Siena, A. Giglio. Servizio di Endoscopia Digestiva, Ospedale Ciaccio, Catanzaro, Italia

Many therapeutical approaches in Helicobacter Pylori eradication have been proposed in the last years. Recently some authors reported in a preliminar study an high incidence of reflux esophagitis in patients after HP eradication therapy [1]. Aim of our study was to evaluate this phenomenon in our endoscopical population during the rast year. Methods: 276 patients affect by peptic ulcer and HP infection were treated with different therapeutic regimens (various antisecretory drugs: omeprazole or ranitidine and various antibiotics: clarytromicine $(\mathrm{A})+$ tinidazole, $\mathrm{A}+$ metronidazole (B), amoxycillin $(C)+$ bismuth, $C+B$. The patients were investigated clinically and endoscopically at 1-6 months after therapy and when dyspeptic symptoms recurred. HP status was assessed by urease test and histology. Results: 169 (61.2\%) were eradicated at 6 months after therapy: 24 of them developed an endoscopically proven reflux esophagitis which was wild (grade I) in all the patients. Conclusions: our study confirms the evidence of reflux esophagitis development in patients treated for HP infection. Such evidence can be explained by different theory: changed eating and drinking habits that can reduce lower esophageal sphincter pressure or the interruption of a chronic therapy with antisecretory drugs for peptic disease. We believe that this phenomenon isn't casual finding in patients without previous evidence of reflux esophagitis and that further studies are needed to clarify this phenomenon.

Reference

[1] Tillenburg B., et al., GUT, Supplement n. 1, Vol. 37, 1995.

\section{C:10 THE ASSOCIATION BETWEEN HELICOBACTER PYLORI AND ISCHAEMIC HEART DISEASE (IHD) ASSESSED BY CORONARY ANGIOGRAPHY}

N. Ossei Gerning, P. Moayyedi ' ${ }^{1}$, A.T.R. Axon ${ }^{1}$, P.J. Grant. Academic Unit of Medicine, Leeds General Infirmary; ${ }^{1}$ Centre for Digestive Diseases, Leeds General Infirmary, Leeds, UK

Introduction: $H$. pylori infection has been reported to increase the risk of IHD. The evidence for this association is conflicting as the methods for evaluating IHD have been imprecise (history and/or ECG changes). We have determined the association of $H$. pylori status with IHD assessed by coronary angiography in a large group of patients. Methods: Consecutive caucasian patients undergoing coronary angiography were recruited. $H$. pylori status was determined by serology (Helico $\mathrm{G}$ ). Coronary angiograms were assessed by an experienced cardiologist who was blinded to $H$. pylori status and IHD was defined as $>50 \%$ stenosis of at least one vessel. Results: 288 patients were recruited (median age 59, age range 33-83 years, 95 female). $204(70 \%)$ patients had IHD and $185(64 \%)$ patients were $H$. pylori positive. $68 \%$ patients with IHD were $H$. pylori positive compared with $50 \%$ without IHD $\left(\lambda^{2} p=0.003\right)$. When adjusted for age, sex, smoking, cholesterol, hypertension and social class $H$. pylori infection was still associated with $\mathrm{IHD}$ (relative risk $=2.4: 95 \% \mathrm{CI}=1.2-5.1 ; \mathrm{p}=$ 0.02).

Conclusion: The study suggests that $H$. pylori is a risk factor for IHD.

\section{C:11 H. PYLORI INFECTION IN PATIENTS WITH AND WITHOUT CORONARY ARTERY DISEASE}

N. Vakil, A. Khurshid, T. Fenske, T. Bajwa. Univ of Wisconsin, Milwaukee WI

The aim of this study was to determine the sero-prevalence of $\mathrm{H}$. pylori infection in patients with suspected ischemic heart disease with normal and abnormal coronary angiograms. Methods: 150 patients undergoing coronary angiography for suspected coronary artery disease were prospectively studied. Angiograms were read by experienced invasive cardiologists blinded to the results of $\mathrm{H}$ Pylori serology, which was determined by a multiwell ELISA assay. Patients with occlusive coronary artery disease were compared to patients with normal coronary arteries (controls) and sub-group analysis was performed in patients with single vessel, two-vessel and three vessel disease. The study was designed to have the statistical power to detect a $20 \%$ difference with a power of $90 \%$ at $p=0.05$. Results: Mean \pm SEM, chi square test or Fisher's exact test for differences between groups. The age of the study population ranged from 37-91 years (Mean = $62 \pm 1) .69$ patients were sero-positive $(46 \%)$. Of 31 patients with normal coronary arteries, $17(55 \%)$ were sero-negative and $14(45 \%)$ were seropositive. Of 119 patients with coronary artery disease, 64 were negative (54\%) and 55 were sero-positive $(46 \% ; P=0.9)$. There were no significant differences between patients with mild moderate and severe disease. 11 of 29 patients (38\%) with single vessel disease were sero-positive. The odds ratios for $H$. pylori infection as a risk factor were not statistically significant: for the presence of coronary artery disease (1.04), for a previous myocardial infarction (1.04), for triple vessel disease $(0.99)$, for severe disease (0.94). Correction for known risk factors did not alter the results. Conclusions: H. pylori infection rates are similar in patients with normal and abnormal coronary arteries, one, two or three vessel disease, mild and severe disease. Odds ratios are similar in men and women with and without previous myocardial infarction. Our data argue against a causal role for $\mathrm{H}$. pylori infection in ischemic heart disease.

\section{C:12 HELICOBACTER PYLORI AND HEART}

M. Caselli ${ }^{1}$, M. Kubbajeh ${ }^{2}$, M. Ruina ${ }^{1}$, P. Gaudenzi ${ }^{1}$, L. Trevisani ${ }^{1}$ M.G. Marangoni ${ }^{1}$, H. Heras Salvat ${ }^{1}$, G.F. Baggioni ${ }^{2}$, V. Alvisi ${ }^{1}{ }^{1}$ School of Gastroenterology, University of Ferrara, Italy; ${ }^{2}$ Institute of Cardiology, Hospital of Comacchio, Italy

H. pylori infection has recently been associated with an increased risk of coronary heart disease (CHD). We studied 83 Italian patients with $\mathrm{CHD}$ and 26 control patients matched for sex and age. We enroled 13 patients with myocardial infarction as evaluated by ECG, typical enzimatic increase and ecocardiography, and 70 ischemic patients as evaluated by coronography in presence of a stenosis of more than $70 \%$ in at least one coronary artery. Exclusion criteria comprised presence of gastritis, peptic ulcer or positive history for dyspeptic symptoms as well. A seropositivity anti-H. pylori by using the Helori-test IgG kit (Eurospital S.p.A.) was detected in 73 (87.9\%) of the ischemic patients in comparison with 12 patients $(46.1 \%)$ in the control group $(p<0.002)$. We do not found any significant difference within the group of ischemic patients neither in relation to the sex or the type of pathology. Successively we have carried out a multivariated analysis in order to evaluate the relative role of different risk factors in our population: $H$. pylori increases the risk from 0.17 to 1.56 , cigarette smoking from 0.50 to 2.20 , familiarity from 0.47 to 1.57 and the social state from 0.11 to 3.73 . A successive multivariated analysis demonstrated that $\mathrm{H}$. pylori plays a role independently from the other risk factors. We conclude that $\mathrm{H}$. pylori should indeed represent a risk factor for $\mathrm{CHD}$ independent from the known ones.

\section{C:13 HELICOBACTER INFECTION AND THE RISK OF MYOCARDIAL INFARCTION}

A. Aromaa ${ }^{1}$, P. Knekt ${ }^{1}$, A. Reunanen ${ }^{1}$, H.I. Rautelin ${ }^{2}$, T.U. Kosunen ${ }^{2}$. ${ }^{1}$ National Public Health Institute and Social Insurance Institution, Helsinki, Finland; ${ }^{2}$ University of Helsinki, Helsinki, Finland

Certain infections including those caused by helicobacters, may increase the risk of cardiovascular diseases. Our purpose was to analyse the association of helicobacter infection with myocardial infarction in the general population.

Methods. A health examination survey was carried out in 12 Finnish cohorts during 1973-76 with a follow-up until the end of 1985. 3471 men, aged 45-64 years, participated in the baseline survey. During the follow-up a new fatal or non-fatal myocardial infarction was observed in 276 subjects who were free of cardiovascular disease at baseline and in 165 subjects who had cardiovascular disease at baseline. A nested case-control study based on these cases and two matched control subjects was performed. A total of 842 controls were matched for age, sex and municipality and cardiovascular disease at baseline. Serum samples taken at baseline were analyzed for helicobacter IgG and IgA antibodies.

Results. 79\% of the subjects with cardiovascular disease at baseline and myocardial infarction during the follow-up had elevated titers of helicobacter IgG antibodies, versus $77 \%$ in their control subjects, which resulted in a relative risk (RR) of 1.16; 95\% confidence interval (CI) 0.73-1.86. Of those who initially did not have cardiovascular disease but had first myocardial infarction during the follow-up, 83\% had IgG antibodies at the baseline, versus $78 \%$ in the control subjects; RR 1.37, (95\% CI 0.94-2.00). Overall IgG positivity of the 441 subjects with myocardial infarction was $82 \%$, versus $78 \%$ in the control subjects, RR 1.29 (95\% CI 0.96-1.73). The respective figures for IgA antibodies were $68 \%$ and $67 \%$, RR 1.05 (95\% CI 0.82-1.34).

Conclusion. The present data indicate a possible although statistically nonsignificant association between the risk of myocardial infarction and 
helicobacter infection as measured by IgG positivity. However, this possible risk seems to be small.

\section{C:14 HELICOBACTER INFECTION IN DEVELOPING ATROPHIC GASTRITIS AMONG SMOKING MEN}

T.U. Kosunen ', P. Sipponen ${ }^{2}$, K. Varis ' , H.I. Rautelin ' ${ }^{\text {, J. Virtamo }}{ }^{3}$, M. Härkönen ${ }^{1}$, O.P. Heinonen ${ }^{1}{ }^{1}{ }^{1}$ University of Helsinki; ${ }^{2}$ Jorvi Hospital, Espoo; ${ }^{3}$ National Public Health Institute, Helsinki, Finland

Active chronic gastritis, known to be caused by helicobacter infection, is followed by atrophic gastritis after a mean of 20 years. Low serum pepsinogen I (PG I) level indicates atrophy in the body of stomach. Our purpose was to study the rate of the association of helicobacter infection with the development of atrophic gastritis.

Methods. Serum samples from 475 smoking men with different levels of PG I at three year intervals were tested for $\mathrm{H}$. pylori IgG and IgA antibodies by enzyme immunoassay. Endoscopies with histological analysis of the biopsies were carried out after the second sample on subjects with gastric complaints and those with low PG I.

Results. Helicobacter antibodies were most common in men with low PG I in second or both samples (95\% and $94 \%$ respectively).

Antibodies were less common in men with persisting normal PG I (79\% in men with gastric symptoms and $66 . \%$ in men selected randomly).

IgA antibodies were most common $(77 \%)$ in men whose pepsinogen I fell below $25 \mathrm{ug} / 1$ during the observation period.

IgG and IgA antibody titers fell most frequently in men with low PG I level at the 3-year follow-up and only $10 \%$ of them were bacteria positive histologically.

Conclusions. Development of atrophic gastritis is regularly associated with helicobacter infection. The progress of atrophic gastritis leads first to the disappearance of detectable bacteria, then to decreasing antibody titers and finally to seronegativity.

\section{C:15 PREVALENCE AND CLINICAL SIGNIFICANCE OF $H$. PYLORI INFECTION IN ELDERLY POPULATION}

T.E. Strandberg, R.S. Tilvis, M. Vuoristo, T.U. Kosunen. University of Helsinki, Helsinki, Finland

Our purpose was to study the association of $\mathrm{H}$. pylori infection with common disease states in randomized age cohorts of elderly subjects of the Helsinki Ageing Study.

Methods. Helsinki Ageing Study is a comprehensive health examination of age cohorts of 75, 80 and 85 years. In 1989-90 456 females and 168 males were examined in general health care using questionaires for symptoms, general diseases (like diabetes, cancer and cardiovascular diseases), use of drugs, interviews, physician's examination and selected laboratory investigations. 624 subjects were studied for helicobacter IgG, IgA and IgM antibodies. 69 subjects were endoscopied for epigastric symptoms.

Results. H. pylori antibodies were found in $68-70 \%$ of the cohorts without differences between sexes. Seropositivity was not associated with common disease states, self-reported history of ulcer disease or the use of non-steroidal anti-inflammatory drugs. Histology was normal in nine subjects; all free of $\mathrm{H}$. pylori infection. Chronic gastritis was found in $87 \%$ of those endoscopied; these included all 20 with verified (serology, histology or culture) infection but also 39 without evidence of the infection. A subject was found to have gastric cancer, but the biopsy was not evaluated for gastritis and bacteria; the subject was seropositive.

Conclusions. Helicobacter infection was very common in elderly general population of Helsinki but in this population it was not associated with an increased risk for any common disease. Chronic gastritis was found in many elderly subjects without detectable $\mathrm{H}$. pylori infection.

\section{C:16 H. PYLORI INFECTION AND CARDIOVASCULAR DISEASES IN ELDERLY POPULATION}

T.E. Strandberg, R.S. Tilvis, M. Vuoristo, T.U. Kosunen. University of Helsinki, Helsinki, Finland

According to some reports, certain infections including those caused by helicobacters, may increase the risk of cardiovascular diseases. Our purpose was to examine the association between $\mathrm{H}$. pylori infection and cardiovascular diseases in a cross-sectional, prospective, population-based study in elderly subjects.

Methods. Sera from 624 female and male citicens of Helsinki, aged 75 , 80 and 85 years were studied for $\mathrm{H}$. pylori IgG, IgA and IgM antibodies at the baseline. All subjects were followed up for five years for patient histories, clinical manifestations, and electrocardiographic or echographic changes related to cardiovascular diseases. Data on total and cardiovascular mortality were based on death certificates.
Results. H. pylori antibodies were found in $68 \%$ of the subjects. Seropositivity was not associated with the occurrence of electrocardiographic or echographic changes and it was not related to well established cardiovascular risk factors except high density lipoprotein cholesterol, which was lower in seropositive subjects. Seropositivity was not related to total or cardiovascular mortality.

Conclusions. Helicobacter antibodies were very common in elderly general population of Helsinki but in this population they were not associated with an increased risk for cardiovascular diseases and mortality.

\section{C:17 LACK OF ASSOCIATION BETWEEN HELICOBACTER PYLORI INFECTION AND ANGIOGRAPHICALLY DOCUMENTED CORONARY HEART DISEASE}

F. Maier, A. Auricchio, M. Nilius, J.-E. Dominguez-Munoz, H. Klein, P. Malfertheiner. Dept. Gastroenterology, Hepatology and Infectious Diseases, Dept. Cardiology; Angiology and Pulmonology,

Otto-von-Guericke-University Magdeburg, Germany

Background: Recent data have linked chronic infection with Helicobacter pylori $(H P)$ to coronary heart disease (CHD). Since symptoms like angina, palpitation or chest pain as well as non-invasive testing are less sensitive and specific for detection of CHD, the gold standard for assessing CHD remains coronary angiography. The aim of this study was to evaluate the incidence of $H P$ infection in patients undergoing routine coronary angiography.

Methods: 257 consecutive patients undergoing routine coronary angiography with known or suspected CHD, valvular defects or electrophysiological abnormalities were screened for antibodies by HP-lgG-ELISA. Laboratory investigations for well known risk factors of CHD, such as arterial hypertension, diabetes mellitus, adipositas, cholesterol, triglycerides uric acid, fibrinogen as well as acute phase reactants (C-reactive protein, ESR, WBC) were performed. Further, smoking history, family history, sex, age and history of gastric symptoms has been investigated. Patients were devided in a group with angiographically documented CHD and in a second group without morphological evidence of CHD. Statistical analysis was performed with unconditional multivariate stepwise logistic regression analysis.

Results: The patient groups were comparable in age and sex. No significant difference was seen in prevalence of $H$. pylori infection and between men and women in patients with and without CHD. Both groups had a comparable mean age (61.88 (CHD+) vs. 58.04 (CHD-). Whereas no significant correlation for $\mathrm{CHD}$ was found with $H P$ infection, multivariable analysis resulted in a very significant correlation with well known risk factors like cholesterol, triglyceride concentration, smoking and age.

Conclusion: The results of our study clearly show that there is no significant link between $\mathrm{CHD}$ and $H$. pylori infection as hypothesized by others.

\section{C:18 CHRONIC URTICARIA AND HELICOBACTER PYLORI} INFECTION

M. Morisset ${ }^{1}$, J.D. de Korwin ' ${ }^{\text {, A. Lozniewski }}{ }^{2}$, G. Kanny ${ }^{1}$, F. Plenat ${ }^{3}$ D.A. Moneret-Vautrin ${ }^{1} .{ }^{1}$ Service de Médecine $D,{ }^{2}$ Laboratoire de Bactériologie, ${ }^{3}$ Laboratoire d'Anatomie Pathologique, Hôpital Central, CHU, 54035 Nancy Cedex, France

The role of gastric pathology in chronic urticaria (CU) has been suspected since the beginning of the century. Recently, the role of Helicobacter pylori $(\mathrm{Hp})$ was pointed out in a few cases. The aim of this retrospective study was to determine the prevalence of $\mathrm{Hp}$ infection in 104 patients (mean age: 37 years) with CU. Seven groups of patients were defined according to the etiology of CU: food allergy (FA) $(n=18)$, false food allergy (FFA) $(n=37)$, physical urticaria (PU) $(n=9)$, abnormal microbial hypersensitivity $(M H)(n=5)$, general diseases (GD) $(n=6)$, autoimmune diseases (AI) $(\mathrm{n}=13)$ and idiopathic urticaria (IU) $(\mathrm{n}=16)$. Hp infection was established by gastric biopsies (urease test, Giemsa staining, culture) in 70 patients and/or by serology (Pyloristat, BioWhittaker) in 53 subjects. Atopy was diagnosed by positive skin prick-tests and total serum IgE level was measured. After age adjustment, there was no significant association of $H p$ infection prevalence with any of the etiologic groups of CU (total: 36\%; FA: $11 \%$; FFA: $46 \%$; PU: $33 \%$; MH: $20 \%$; GD: $67 \%$; AI: $31 \%$; IU: $38 \%)(p=0.23)$. Hp infection was not correlated with serum IgE levels, and atopy was correlated with the absence of $H p$ infection $(p=0.006)$ This study did not find any relation between $H p$ infection and main $\mathrm{CU}$ categories. 


\section{C:19 H. PYLORI (HP) INFECTION AND TRANSAMINASES}

N. Figura, C. Gennari. Inst. of Internal Medicine, University of Siena, Siena, Italy

The production of granulating toxin (GT) by Helicobacter hepaticus has been associated with the development of hepatitis and hepatic tumours in mice. $H P$ also produces GT, in addition to the vacuolating toxin called VacA which is mostly expressed together with the cytotoxin-associated protein CagA. However, no association has yet been made between $H P$ infection and liver damage. Patients and Methods. Levels of glutamic oxaloacetic and glutamic pyruvic transaminases (GOT, GPT) in serum samples of $38 H P$ positive adult patients were retrospectively compared with those of $23 H P$ negative age-matched controls. HP positive patients were also tested for serum antibodies to CagA by Western blotting. All patients had a negative history of liver and muscular diseases, were negative for markers of virus hepatitis A, B, and C, and did not take hepatotoxic drugs. Results. Mean levels \pm SD (IU/L) of serum GOT and GPT were $22.61 \pm 9.54$ and $21.82 \pm 11.09$ in infected patients, and $23.57 \pm 14.90$ and $19.78 \pm 10.57$ in uninfected patients (non significant). GOT and GPT levels in the $19 \mathrm{CagA}^{+}$infected patients were $25.63 \pm 11.75$ and $24.37 \pm$ 13.35 versus $19.58 \pm 5.44(\mathrm{P}=0.049$, significant $)$ and $19.26 \pm 7.81(P$ $=0.158$, non-significant) in the $19 \mathrm{CagA}^{-}$infected patients. Conclusions. GT, if produced in vivo, does not seem to injure the liver. Persons with $\mathrm{CagA}^{+} H P$ infection might have a mild degree of liver damage. The $H P$ vacuolating toxin, should it reach the liver, could alter cell permeability and/or influence cell growth factors and injure the hepatocytes. Increased GOT levels, however, could also derive from gastric epithelial cells which in $\mathrm{CagA}^{+}$patients seem more damaged.

\section{C:20 FOOD ALLERGY AND H. PYLORI INFECTION}

N. Figura ${ }^{1}$, A. Perrone ${ }^{2}$, C. Gennari ${ }^{1}$, M. Vagliasindi ${ }^{2}$, P Rottoli ${ }^{2}{ }^{1}{ }^{1}$ Int. Medicine Insts., Siena Univ., Italy; ${ }^{2}$ Resp. Diseases Insts., Siena Univ., Italy

Most antigens get to the immune system through mucosae. Gastrointestinal mucosa is a barrier for alimentary antigens. Inflammatory processes could alter the integrity of the barrier, increase the permeability of mucosae, and enhance crossing of food antigens. Many $H$. pylori $(H P)$ infected persons test positive for basophil-bound and serum specific IgE. As HP can also induce IgE-mediated histamine release from human basophils in vitro and mucosal IgE positive mast cells are involved in food allergy (FA), we investigated whether $H P$ infection could be associated with FA. 38 adult patients with symptomatic FA and 53 age-matched controls (31 patients with respiratory allergy and 22 healthy subjects) were studied. Allergies were determined by titrating $\operatorname{IgE}$ to alimentary or respiratory antigens using Pharmacia CAP System RAST FEIA. HP infection was diagnosed serologically by an ELISA. Anti-CagA antibodies were investigated in $H P$ positive persons by Western blotting. Results:

\begin{tabular}{lll}
\hline Patient group & $H P$ positive & CagA positive \\
\hline Food allergy & $42.1 \%$ & $62.5 \%$ \\
Respiratory allergy & $45.1 \%$ & $28.5 \%$ \\
No allergy & $47.8 \%$ & $27.2 \%$ \\
\hline
\end{tabular}

CagA seropositivity in FA infected patients was significantly more prevalent than in infected controls $(P=0.030$, Mantel-Haenszel test). We postulate that serious inflammatory gastric mucosal lesions could enhance the epithelial permeability and render non-selective the passage of allergens which, in atopic persons, could directly stimulate an IgE response. CagA ${ }^{+}$ $H P$ infection could trigger or worsen FA.

\section{C:21 GALLSTONES AND H. PYLORI (HP) INFECTION}

N. Figura ${ }^{1}$, D. Vaira ${ }^{2}$, R. Vernillo ${ }^{1}$, J.E. Crabtree ${ }^{3}$, F. Bazzoli ${ }^{2}{ }^{1}$ Univ. of Siena; ${ }^{2}$ Univ. of Bologna, Italy; ${ }^{3}$ Univ. of Leeds, UK

Gallstones consist of cholesterol crystals and calcium salts. Several proteins, including immunoglobulins (Ig), have been reported to have cholesterol cristallisation-promoting activity. In addition, accumulation of bacterial products in bile can be associated with hydrolysis of bilirubin conjugates and precipitation of insoluble organic calcium salts. HP infection and gallstones are conditions very common and the prevalence of both increases with age. Their possible association has never been considered. IgG and IgA to $H P$ were investigated by Western blotting (WB) in serum samples and in bile samples obtained at cholecystectomy from 15 patients with gallstone disease (GD) at 1:200 and 1:50 dilutions, respectively. The presence of $H P$ antigens in bile was investigated by WB: proteins were separated by electrophoresis in polyacrylamide gel and transferred onto nitrocellulose which was blotted with antibodies to CagA, urease, and whole $H P$ antigens (provided by Biocine, Siena). Ten patients were seropositive for $H P$ infection; 7 patients also had serum antibodies to CagA. Nine bile samples from infected patients tested positive for anti-HP IgA. No bile sample from uninfected patients contained anti- $H P$ IgA. Immune reactivity to $H P$ antigens of IgA in bile and IgG in serum was similar. Two bile samples from CagA seropositive patients contained an antigen of $60 \mathrm{kDa}$ $c a$. which immunoreacted solely with the anti-CagA antibody.

Conclusions. Bile samples from HP seropositive GD patients contain $H P$ antigens and related antibodies. Biochemical characterisation and evaluation of potential nucleating (or antinucleating) activity of these proteins may help in understanding if $H P$ infection has a role in the pathogenesis of GD.

\section{C:22 H. PYLORI (HP) INFECTION AND THYROID DISEASES}

N. Figura, E. Guarino, A. Gragnoli, G. Di Cairano, F. Lorè, D. Cataldo, C. Gennari. Institute of Internal Medicine, University of Siena, Siena, Italy

Thyroid diseases (TD) are frequently associated with the production of autoantibodies to components or products of the thyroid gland like colloid and peroxidase. As $H P$ infection can stimulate an autoimmune response, we investigated the possible role of $H P$ infection and of infection by $H P$ strains which express the cytotoxin-associated protein CagA in the development of TD. A group of 48 women with TD and autoantibodies to thyroglobulin (mean age 48.81 \pm 15.92 years) and 33 control women (mean age $48.17 \pm$ 16.06) were examined for $H P$ infection and for anti-CagA serum IgG by Western blot. Serum samples were diluted 1:200 and blotted with a HP cell suspension (previously denatured in Laemmli) run electrophoretically in a polyacrylamide gel with sodium dodecyl sulphate, and transferred onto nitrocellulose. Tests were considered positive for infection in the presence of 5 or more bands of reaction. Results. Thirty-four TD patients $(68.7 \%)$ tested seropositive for $H P$ infection vs. 16 controls $(48.4 \% ; P=0.043$, Mantel-Haenszel test). The prevalence of IgG to HP CagA, UreB, and HSP in infected patients with TD was $70.5 \%, 58.8 \%$, and $55.8 \%$ vs. $50.3 \%$ $(P=0.16), 50.0 \%$ and $31.2 \%(P=0.107)$ in infected controls. Levels of autoantibodies to thyroglobulin in $H P$ positive and negative TD patients were similar $(P=0.747)$, as well as levels of TSH $(P=0.950)$ and $\mathrm{FT}_{3}$ $(P=0.751)$. Conclusions. The association observed between TD and $H P$ infection deserves further investigation. Comparisons between levels of autoantibodies to thyroglobulin before and after bacteriological eradication could help to assess a possible role of $H P$ infection in thyroid diseases.

\section{C:23 IS HELICOBACTER PYLORI (HP) INFECTION ASSOCIATED WITH A TENDENCY TOWARDS A PROCOAGULANT STATE IN HEALTHY INDIVIDUALS?}

F. Parente, V. Imbesi, G. Maconi, E. Rossi ${ }^{1}$, G. Bianchi Porro. Department of Gastroenterology, ${ }^{1}$ Department of Haematology, L. Sacco University Hospital, Milan, Italy

The influence of HP on circulating levels of coagulation factors related to ischaemic heart disease (IHD) remains uncertain. The aim of this study was, therefore, to investigate the possibility for HP to induce a tendency towards a procoagulant state, thereby influencing the risk of IHD. 368 consecutive asymptomatic blood donors were initially admitted to the study. Exclusion criteria included: age $>51$ years, recent intake of any compound capable of interfering with blood coagulation, use of oral contraceptives, previous HP eradication therapy, diagnosis of IHD, peptic ulcer or any systemic chronic illness. 300 subjects ( 229 males), aged $20-51$ years (mean 34.7 ) fulfilled the above criteria and were enrolled into the study. They were questioned about past medical history, diet, alcohol and cigarette consumption, education and socio-economic status. Their blood was analyzed for total and HDL cholesterol, ESR, C reactive protein, fibrinogen, PT, PTT, factor VII:C, haemoglobin, platelet and leucocyte count. In addition, prothrombin cleavage fragment $(\mathrm{F} 1+2)$, which is an index of prothrombin activation, was assayed. HP status was then determined in all subjects by means of a specific IgG antibodies assay. The overall prevalence of HP infection was 53\% (158/300); as expected, HP-positive subjects were significantly older $(37.0+7.9)$ than HP-negative group $(32.2+7.4)$. The two groups did not significantly differ as for alcohol and cigarette consumption, total and HDL cholesterol, plasma fibrinogen, total leucocyte and platelet count, PT and PTT, ESR and C-reactive protein. In contrast, F VII:C and F1 $+2^{*}$ levels were significantly higher in HP-positive than HP-negative subjects $\left({ }^{*} 1.0+\right.$ 0.4 vs $0.89+0.3$ ); however, after adjustment by multiple regression for age, sex, socio-economic status and smoking habits there was no longer any evidence of a significant association between HP and these factors. In conclusion, we found no evidence in healthy individuals to support the hypothesis that HP infection is associated with a tendency towards a procoagulant state. 
1C:24 PREVALENCE OF H. PYLORI (HP) INFECTION AND HP-RELATED UPPER GI LESIONS IN PATIENTS WITH INFLAMMATORY BOWEL DISEASES (IBD). A CASE-CONTROL STUDY

F. Parente, P. Molteni, S. Bollani, G. Maconi, B. Rembacken ', A. Axon ${ }^{1}$, G. Bianchi Porro. GI Units, L. Sacco Hospital, Milan, Italy; ' LGI, Leeds, UK

Although a reduced prevalence of HP infection has been reported in IBD patients (pts), the clinical significance of HP infection in this setting remains unknown. The aim of this study was, therefore, to evaluate the prevalence of HP infection in a large series of IBD pts as well as the frequency of gastroduodenal lesions in those accepting to undergo upper GI endoscopy.

216 consecutive IBD pts [122 with Crohn's disease (CD) and 94 with ulcerative colitis (UC)] had their anti-HP IgG titres measured using a commercial ELISA kit. 216 blood donors matched for age, sex, place of birth in Italy and socio-economic status served as controls. All pts were offered the possibility of undergoing upper GI endoscopy. The overall seroprevalence of HP infection was $48 \%$ in IBD pts vs $59 \%$ in the control group ( $\mathrm{p}$ $<0.01$ ), with a significantly lower frequency in CD vs UC pts $(41 \%$ vs $56 \%$ ). After adjustment for age, education and socio-economic status CD remained associated with a significantly lower risk of HP infection. No association was shown between reduced HP seropositivity and previous rx with SASP, 5-ASA, steroids or azathioprine, whereas a weak relationship between previous antimicrobial $\mathbf{r x}$ and lower risk of HP infection was found. 179 pts (106 with CD and 73 with UC) underwent endoscopy; the prevalence of peptic ulcer was similar in both groups (4.7\% in CD and 5.5\% in UC pts), however, 11 more CD pts had ulcers which were interpreted as CD-related. 7 of them had a history of foregut complaints but the remaining had never suffered from dyspepsia. $64 \%$ of these $C D$-associated gastroduodenal ulcers were HP -ive. Our findings show that CD but not UC pts have a reduced prevalence of HP infection as compared to matched healthy controls. This is partly due to a greater use of antimicrobials. $10 \%$ of $\mathrm{CD}$ pts have also a gastroduodenal localisation of their disease, which is often asymptomatic. Most of these CD-associated ulcers are unrelated to HP infection.

\section{C:25 FIBRINOGEN AND H. PYLORI IN ASYMPTOMATIC POST MI} PATIENTS AND HEALTHY CONTROLS

J. Rajput-Williams ' , N.R. Williams ' ${ }^{1}$, P.G. Johnson ${ }^{2}$, R.J. Dickinson ${ }^{3}$.

${ }^{1}$ Papworth Hospital, Cambridge, UK; ${ }^{2}$ BSIA, Brentford, UK;

${ }^{3}$ Hinchingbrooke Hospital, Huntingdon, $U K$

A link between $H$. pylori seropositivity and coronary heart disease via plasma fibrinogen has been suggested, although this has been disputed.

Fibrinogen (and Factor VII) were measured in non-smoking men who recently had a myocardial infarction (MI; $n=35$ ) and healthy controls ( $=27$ ) with known $H$. pylori status (assessed by carbon-13 urea breath test, BSIA, UK). Results are presented in the Table.

Table. Plasma fibrinogen. Results are mean (95\% confidence interval). Statistical significance was assessed by t-test. Pairs of comparisons are marked $\mathrm{a}, \mathrm{b}$ and $\mathrm{c}$.

\begin{tabular}{llrll}
\hline & H. pylori & \multicolumn{1}{c}{ n } & Fibrinogen (g/) & \\
\hline Control & Negative & 20 & $2.68(2.48-2.88)$ & a, b, c \\
Control & Positive & 7 & $3.07(2.85-3.30)$ & a \\
Post-MI & Negative & 27 & $3.04(2.82-3.26)$ & b \\
Post-MI & Positive & 8 & $3.26(2.64-3.89)$ & c \\
\hline
\end{tabular}

a, $P=0.044 ; b, P=0.026 ; c, P=0.010$

There were no significant differences in Factor VII (data not shown). In conclusion, this data supports the hypothesis of an association between $H$. pylori status and fibrinogen.

\section{C:26 LOW PREVALENCE OF HELICOBACTER PYLORI INFECTION IN PATIENTS WITH CROHN'S DISEASE IRRESPECTIVE OF SULPHASALAZINE THERAPY}

T. Kamada ${ }^{1}$, K. Haruma ' , M. Mihara ${ }^{1}$, T. Goto ${ }^{1}$, K. Kiyohira ${ }^{1}$ H. Kawaguchi ${ }^{1}$, M. Ito ${ }^{1}$, K. Sumii $^{1}$, G. Kagiyama ${ }^{1}$, N. Uemura ${ }^{2}$ ' $^{1}$ First Dept, of Internal Medicine, Hiroshima University School of Medicine, Hiroshima, Japan; ${ }^{2}$ Division of Gastroenterology, Kure Kyousai Hospital, Hiroshima, Japan

Recent reports have shown that longterm therapy with sulphasalazine leads to eradication of Hericobacter Pylori (Hp) infection in patients with inflammatory bowel disease. The AIM of this study was to investigate the prevalence of $\mathrm{Hp}$ infection in patients with Crohn's disease in comparison with sex-and age-matched controls, along with serum gastrin (G) and pepsinogens (PGs).
Methods: This study consisted of 46 patients with Crohn's disease (31 $\mathrm{M}, 15 \mathrm{~F}$; mean age $25.5 \mathrm{yr}$ ) and 92 controls $(62 \mathrm{M}, 30 \mathrm{~F}$; mean age 25.4 yr) having no localized lesion in upper GI tract. Endoscopic biopsies were obtained from the antrum (2bx) and fundus (2bx). The presence of $\mathrm{Hp}$ infection was judged by Giemsa stain. Serum $G$ and PGs were determined by RIA. Values were expressed as mean $\pm \mathrm{SE}$.

Results: The overall prevalence of Hp infection in patients with Crohn's disease was $4.4 \%(2 / 46)$ which was significantly less than that of $26.1 \%$ $(24 / 92)$ in the controls $(\mathrm{p}<0.01)$. The prevalence of $\mathrm{Hp}$ infection was $4.8 \%(1 / 21)$ in patients with Crohn's disease currently or previously on sulpfasalazine therapy and $4 \%(1 / 25)$ in those who had never had sulphasalazine therapy. There was no difference in prevalence between the two groups. Serum $\mathrm{G}$ was higher in patients with Crohn's disease than in controls, but no significant difference was found $(71.7 \pm 6.5 \mathrm{pg} / \mathrm{ml}$ vs. 88.7 $\pm 5.3 \mathrm{pg} / \mathrm{ml}, \mathrm{p}=0.204$ ). Serum PGI, PGII, and PGI/II ratio were $45.2 \pm$ $4.1 \mathrm{ng} / \mathrm{ml}, 8.65 \pm 1.2 \mathrm{ng} / \mathrm{ml}, 5.72 \pm 0.48$ in patients with Crohn's disease and $43.8 \pm 2.2 \mathrm{ng} / \mathrm{ml}, 10.4 \pm 1.0 \mathrm{ng} / \mathrm{ml}, 5.43 \pm 0.19$, respectively. There was no significant difference between the two groups.

Conclusion: Hp infection is extremely low in patients with Crohn's disease irrespective of sulphasalazin therapy.

\section{C:27 LOW PREVALENCE OF HELICOBACTER PYLORI INFECTION IN PATIENTS WITH REFLUX ESOPHAGITIS}

M. Mihara, K. Haruma, T. Kamada, K. Kiyohira, T. Goto, M. Sumii, S. Tanaka, M. Yoshihara, K. Sumii, G. Kajiyama. First Dept. of Internal Medicine, Hiroshima University School of Medicine, Hiroshima, Japan

Recent studies have shown the close association of H. pylori (HP) infection with gastritis or peptic ulcer diseases, but there is little information concerning the relationship Hp infection and reflux esophagitis (RE). The Aim of this study is to investigate the prevalence of HP in patients with RE, along with histologic gastritis. Methods: The study population consisted of 70 patients with RE and 70 sex- and age-matched controls who have no localized lesions in the upper GI tract. Hp infection was evaluated by Giemsa stain on antral and fundic biopsies and serum IgG antibody. Gastritis and atrophy were scored as follows: normal $=0$, mild $=1$, moderate $=2$ and severe $=3$. Serum pepsinogens $($ PGs) were determined by RIA

Results: (Mean $\pm \mathrm{SE}, * \mathrm{p}<0.05, * * \mathrm{p}<0.01)$

\begin{tabular}{lll}
\hline & Controls & RE \\
\hline M/F & $49 / 21$ & $49 / 21$ \\
Mean age & 59.8 & 59.8 \\
Hp positive (\%) & $47(67)$ & $26(37)^{* *}$ \\
Gastritis score & $1.48 \pm 0.11$ & $0.55 \pm 0.14^{* *}$ \\
Atrophic score & $1.28 \pm 0.13$ & $0.28 \pm 0.1^{* *}$ \\
PGI & $47.5 \pm 3.9$ & $62.2 \pm 4.7^{*}$ \\
PGII & $16.9 \pm 1.6$ & $11.9 \pm 1.6$ \\
VII ratio & $3.3 \pm 0.3$ & $5.7 \pm 0.5 * *$ \\
\hline
\end{tabular}

Conclusions: HP infection is significantly lower in patients with RE than in controls. The pathogenesis of RE may be related to low prevalence of atrophic gastritis (preservation of acid secretion) in this patients group.

\section{C:28 PREVALENCE OF H. PYLORI (HP) INFECTION IN INFLAMMATORY BOWEL DISEASE (IBD) -A CONTROLLED STUDY}

L.S. Sousa, A.M. Santos, T.C. Macedo, A.S. Pinto, A.C. Sousa, J. Reis, S. Vá, M.G. Quina. Hosp. Pulido Valente, Lisbon, Portugal

Objectives: To evaluate Hp prevalence in patients (p.) with IBD, submitted to several therapeutical regimens. Material \& Methods: $50 \mathrm{p}$. were studied prospectively. 26 with Crohn Disease $(C D)$, median age $=44.82 \pm 15.72$; 24 with Ulcerative Colitis (UC), median age $=44.52 \pm 12.84$. Results were compared to those obtained in $\mathbf{5 0}$ healthy volunteers studied as control group (CG). These groups were matched by age ( $p<0.0042$ between $C D$ and UC groups and $p<0.05$ between the two pathological groups and CG. Hp was evaluated as follows: 1) Urea ${ }^{13} \mathrm{C}$ Breath Test ( $n=43$ p.+ 50 CG); 2) Urease \& Histology of gastric mucosae in dispeptic p. $(\mathrm{n}=11)$. Statistic analysis was carried out by $\chi^{2}$, Kendall Coefficient of Concordance and Spearman Rank Order Correlation. Results: Prevalence of $\mathrm{Hp}$ was as follows: IBD group $=58 \%(\mathrm{CD}=42 \%$ and UC $=75 \%$ with $\mathrm{p}=0.01$ ) and $\mathrm{CG}=62 \%$. A significant decrease of $\mathrm{Hp}+$ prevalence was verified in CD p. treated with Metronidazol (MTN) $(40 \%, p<0.02)$ and in those submitted to surgery $(31 \%, p=0.008)$, also dominantly ( $87.5 \%$ of operated p.) included in the CD population. Duration, extension, clinical activity or other therapies (sulphasalazine, mesalazine, steroids and azathioprine) were not correlated to the Hp status in the IBD group. Conclusions: 1. Prevalence of $\mathrm{Hp}$ infection was similar in IBD population and control group. A significant decrease was shown in CD when compared to UC group. 2. Low prevalence of $\mathrm{Hp}+$ in the $\mathrm{CD}$ group was associated with previous surgery and with present or previous MTN therapy. Almost 
all patients submitted to surgery had medical intractability after being submitted to intensive drug treatment.

\section{C:29 DOES HELICOBACTER PYLORI AFFECT THE BLOOD AMMONIA LEVEL OF HEMODIALYSIS PATIENTS?}

Z. Fireman, D. Coscas, A. Sternberg, Y. Wagner, H. Zonder. Hillel Yaffe Medical Center, Hadera, Israel

Hyperammonemia, alone, causes mental state changes and, in combination with other substances, can lead to a comatose state. Helicobacter pylori (HP) through its urease activity produces ammonia from urea. The hypothesis is that urea, which is found in large quantities in the gastric lumen of uraemic patients, is transformed into ammonia, by the HP urease activity which is transmitted to the blood, contributing to mental state changes in these patients

The blood ammonia levels of HP positive uraemic patients were compared with those of HP negative patients. Sixteen patients on chronic hemodialysis were evaluated. The examinations were carried out prior to the hemodialysis sessions. Nine patients were found to be $\mathrm{HP}(+)$ and seven HP(-).

\begin{tabular}{|c|c|c|c|c|c|}
\hline & \multirow[t]{2}{*}{ No. of pts } & \multicolumn{2}{|l|}{ Stomach } & \multicolumn{2}{|l|}{ Blood } \\
\hline & & $\begin{array}{l}\text { urea } \\
\text { (mg/dL) }\end{array}$ & $\begin{array}{l}\text { ammonia } \\
(\mu \mathrm{g} / \mathrm{dL})\end{array}$ & $\begin{array}{l}\text { urea } \\
\text { (mg/dL) }\end{array}$ & $\begin{array}{l}\text { ammonia } \\
(\mu \mathrm{g} / \mathrm{dL})\end{array}$ \\
\hline $\begin{array}{l}\mathrm{HP}+ \\
\mathrm{HP}- \\
\mathrm{P} \text { value }\end{array}$ & $\begin{array}{l}9 \\
7\end{array}$ & $\begin{array}{l}33.1 \\
26.5 \\
0.499\end{array}$ & $\begin{array}{l}457.1 \\
475.3 \\
0.5375\end{array}$ & $\begin{array}{l}58.9 \\
53.7 \\
0.5736\end{array}$ & $\begin{array}{l}51.1 \\
63.4 \\
0.125\end{array}$ \\
\hline
\end{tabular}

Conclusion:

1. No difference was found between the blood ammonia levels in HP positive and HP negative patients.

2. Although ammonia is elevated in the gastric lumen of both $\mathrm{HP}(+)$ and HP(-) patients, its blood level is normal.

3. Hyperammonemia is probably not a contributing factor to mental changes in uraemic patients.

4. There is no clear-cut explanation for the elevated gastric ammonia level in HP negative patients.

\section{C:30 HIGH PREVALENCE OF HELICOBACTER PYLORI INFECTION IN CORONARY HEART DISEASE} DEMONSTRATED BY THE ${ }^{13}$ C-UREA BREATH TEST

C. Martín de Argila, D. Boixeda, A. Fuertes, R. Cantón, M. Barba, C. Arocena, J.P. Gisbert. García Plaza, "Ramón y Cajal" Hospital, Madrid, Spain

Previous studies have reported an association between coronary heart disease (CHD) and $H$. pylori infection, but all of them have used serological tests to confirm infection.
Aim: To determine the prevalence of $H$. pylori infection in a large group patients with $\mathrm{CHD}$ and its relationship with different conventional $\mathrm{CHD}$ risk factors.

Materials and Methods: One hundred and twelve consecutive patients (95 males and 17 females, Mean age: $59.1 \pm 11.9 \mathrm{yrs}$ ) were studied with documented CHD admitted at the Coronary Care Unit in our Hospital. Patients with previous history of peptic ulcer disease or digestive conditions were excluded. Information was inquired on the presence of conventional risk factors for cardiovascular disease (diabetes, hyperlipidemia, smoking, and arterial hypertension). Eighty-three healthy persons ( 24 males and 59 females; mean age: $51.5 \pm 10.7$ yrs) comprised the control group. IgG antibodies to $H$. pylori were measured in all persons by means of a serological ELISA method (Helico-G, Porton, Cambridge, UK). All persons with CHD underwent also a ${ }^{13} \mathrm{C}$-urea breath test $\left({ }^{13} \mathrm{C}\right.$-UBT $)$ to study $H$. pylori infection.

Results: Ninety-one (81.3\%) of 112 patients with $\mathrm{CHD}$ had a positive serology for $H$. pylori ( $>10 \mathrm{U} / \mathrm{ml}$ ) compared with $53(63.8 \%)$ persons out of the 83 in the control group ( $p<0.01$ ). In 90/112 (80.4\%) of patients with $\mathrm{CHD}$ the ${ }^{13} \mathrm{C}$-UBT was positive $\left(\delta \mathrm{CO}_{2}>5 / 1000\right)$. No association was observed between $H$. pylori infection and the different risk factors for CHD:

\begin{tabular}{llllll}
\hline & Yes & & No & \\
\cline { 2 - 3 } Characteristic & H. pylori + & H. pylori & & H. pylori + & H. pylori - \\
\hline History of: & & & & & \\
Smoking & $43(82.7)$ & $9(17.3)$ & & $47(78.3)$ & $13(21.7)$ \\
Hyperlipidemia & $33(76.7)$ & $10(23.3)$ & & $57(82.6)$ & $12(17.4)$ \\
Diabetes & $19(82.6)$ & $4(17.4)$ & $71(79.8)$ & $18(20.2)$ \\
Hypertension & $40(76.9)$ & $12(23)$ & $50(83.3)$ & $10(16.7)$ \\
\hline
\end{tabular}

$p>0.05$ in all comparisons between the different groups.

Conclusions: Patients with CHD had a high prevalence of $H$. pylori infection, significantly higher than that observed among healthy persons. The absence of an association between conventional risk factors for CHD and $H$. pylori infection suggests an independent action of $H$. pylori in its possible involvement in CHD. 